\title{
SERUM LIPID ANOMALIES IN ADULT PATIENTS WITH ACUTE ISCHEMIC STROKE
}

\author{
Daniela Arabadzhieva ${ }^{1}$, Zdravko Slavov ${ }^{2}$, Ara Kaprelyan ${ }^{1}$ \\ ${ }^{1}$ Department of Neurology and Neurosciences, Medical University of Varna \\ ${ }^{2}$ Department of Informatics, Chernorizets Hrabar Varna Free University, Varna
}

\begin{abstract}
INTRODUCTION: There is rising evidence of the role of hyperlipidemia and dyslipidemia in the development of acute ischemic stroke (AIS). There is a considerable risk for AIS in adult individuals with these abnormalities. Our purpose was to reveal the incidence rate of the disorders of lipid metabolism in adult AIS patients.

MATERIAL AND METHODS: We examined 129 male and 129 female patients with AIS at a mean age of 70.59 \pm 7.22 years hospitalized in the First Clinic of Neurology, St. Marina University Hospital of Varna in 2007-2013. The serum levels of total cholesterol, triglycerides, HDL- and LDL-cholesterol at admission were examined. Statistical data processing was performed by ANOVA as $t$-criterion was considered significant if $\mathbf{p}<0.05$.

RESULTS: The mean values of total cholesterol and LDL-cholesterol were slightly elevated while those of triglycerides were slightly reduced. The mean values of total cholesterol and triglycerides were higher in males than in females. Total cholesterol values were considerably more commonly higher in females than in males as the mean total cholesterol concentrations were higher in the female patients with normal, decreased and increased levels of this parameter. LDL-cholesterol was more commonly elevated as its mean values were higher in the female patients with its normal, decreased and increased levels.

CONCLUSION: A regular control of the parameters of lipid metabolism and keeping-up the healthy lifestyle in adult individuals and in patients with cardiovascular diseases could ensure a successful prevention of ischemic stroke.
\end{abstract}

Keywords: acute ischemic stroke; total cholesterol; triglycerides; HDL-Cholesterol, LDL-Cholesterol

Address for correspondence:

Daniela Arabadzhieva, $M D, P h D$

First Multiprofile Clinic of Neurology,

St. Marina University Hospital of Varna,

Medical University of Varna

1 Hristo Smirnenski Str.

9010 Varna, Bulgaria

e-mail:d.arabadzhieva@abv.bg

Received: March 03, 2015

Accepted: June 10, 2015

\section{INTRODUCTION}

Acute ischemic stroke (AIS) is the most common cerebrovascular disease worldwide. Numerous recent publications deal with the role of disorders of the lipid metabolism in the prognosis (1-6) of AIS. Meanwhile, a variety of risk factors for AIS has been identified. Here belong the common disturbances of lipid metabolism which are considerably widespread among the young and, especially, among adult Bul-

Scripta Scientifica Medica, vol. 47, No 2, 2015, pp. 31-36

Copyright (c) Medical University of Varna 
garian population, too. That is why effective and timely prevention of AIS is of rising socio-medical importance.

Our purpose was to reveal the incidence rate of the abnormalities of some routine parameters of lipid metabolism in adult male and female AIS patients.

\section{MATERIAL AND METHODS}

We examined 129 male and 129 female patients with AIS at a mean age of $70.59 \pm 7.22$ years hospitalized in the First Clinic of Neurology, St. Marina University Hospital of Varna in 2007-2013. The serum levels of total cholesterol, triglycerides, HDLand LDL-cholesterol at admission were examined. Statistical data processing was performed by using of ANOVA as $t$-criterion was considered significant if $\mathrm{p}<0.05$. SPSS, version 13.0 software was applied.

\section{RESULTS}

Our results illustrate the role of several lipid metabolism-related risk factors for AIS etiopathogenesis in adulthood.

The mean serum levels of these parameters of lipid metabolism such as total cholesterol, triglycerides, HDL- cholesterol and LDL-cholesterol in AIS patients are illustrated on Table 1 through Table 3 as well as on Figure 1 through Figure 4.
The mean values of total cholesterol and LDLcholesterol are slightly elevated while those of triglycerides are slightly reduced. The mean values of total cholesterol and triglycerides are higher in males than in females. The analysis of the number and relative share of the patients with normal, decreased and increased values of these lipid parameters reveals the frequency of their anomalies and thus illustrates the importance of their regular examinations under outpatient conditions for preventive purposes.

The number of our AIS patients with normal total cholesterol (Figure 1) and HDL-cholesterol concentrations (Figure 3) prevails. Only a few patients present with very low or very high HDL-cholesterol levels (Figure 3). In most AIS patients, there are moderately to considerably reduced triglyceride values (Figure 2). The number of the patients with normal LDL-cholesterol concentrations is similar to that of the patients with moderately elevated LDL-cholesterol values (Figure 4). There are, however, single AIS patients with considerably increased levels of this parameter, too (Figure 4).

Total cholesterol values are considerably more often higher in females than in males as the mean total cholesterol concentrations are higher in the female patients with normal, decreased and increased levels of this parameter. LDL-cholesterol is more common-

Table 1. Mean serum lipid values in adult AIS patients at admission

\begin{tabular}{|c|c|c|c|}
\hline \multirow{2}{*}{ Serum lipids } & \multicolumn{3}{|c|}{ Mean values (mmol/L) } \\
\hline & males & females & total \\
\hline total cholesterol $(n=257)$ & $5.11 \pm 1.57$ & $5.51 \pm 1.32$ & $5.31 \pm 1.46$ \\
\hline triglycerides $(n=256)$ & $1.88 \pm 1.26$ & $1.99 \pm 1.41$ & $1.94 \pm 1.34$ \\
\hline HDL-cholesterol $(n=234)$ & $1.34 \pm 0.50$ & $1.33 \pm 0.46$ & $1.34 \pm 0.48$ \\
\hline LDL-cholesterol $(\mathrm{n}=235)$ & $2.47 \pm 1.24$ & $2.81 \pm 1.22$ & $2.64 \pm 1.24$ \\
\hline
\end{tabular}

Table 2. Mean normal and pathological serum lipid values in adult AIS patients at admission

\begin{tabular}{l|c|c|c|c|c|c|}
\hline \multirow{2}{*}{$\begin{array}{l}\text { Serum lipids } \\
\text { (mmol/L) }\end{array}$} & \multicolumn{2}{c}{$\begin{array}{c}\text { patients with } \\
\text { normal values }\end{array}$} & \multicolumn{2}{c}{ patients with increased } & \multicolumn{2}{c|}{$\begin{array}{c}\text { patients with } \\
\text { decreased values }\end{array}$} \\
\cline { 2 - 7 } & males & females & males & females & males & females \\
total cholesterol & $4.30 \pm 0.62$ & $4.38 \pm 0.58$ & $6.61 \pm 1.50$ & $6.37 \pm 0,94$ & $2.32 \pm 0.28$ & $1.33 \pm 0.0$ \\
triglycerides & $2.70 \pm 0.10$ & $2.69 \pm 0.09$ & $4.21 \pm 1.35$ & $3.96 \pm 2,25$ & $1.38 \pm 0.54$ & $1.51 \pm 0.52$ \\
HDL-cholesterol & $1.25 \pm 0.15$ & $1.27 \pm 0.17$ & $2.07 \pm 0.50$ & $2.02 \pm 0,61$ & $0.84 \pm 0.12$ & $0.79 \pm 0.16$ \\
LDL-cholesterol & $1.65 \pm 0.49$ & $1.83 \pm 0.51$ & $3.65 \pm 1.00$ & $3.78 \pm 0,89$ & - & - \\
\hline
\end{tabular}


Daniela Arabadzhieva, Zdravko Slavov, Ara Kaprelyan

Table 3. Percentage of adult AIS patients with pathological serum lipid values at admission

\begin{tabular}{|l|c|c|c|c|c|c|}
\hline \multirow{2}{*}{ Serum lipids (mmol/L) } & \multicolumn{3}{|c|}{ \% of patients with increased values } & \multicolumn{3}{c|}{ \% of patients with decreased values } \\
\cline { 2 - 7 } & males & females & total & males & females & total \\
total cholesterol & 36.43 & 58.14 & 47.29 & 2.33 & 0.78 & 1.55 \\
triglycerides & 5.43 & 17.83 & 11.63 & 15.50 & 77.52 & 46.51 \\
HDL-cholesterol & 20.93 & 15.50 & 18.22 & 20.93 & 12.40 & 16.66 \\
LDL-cholesterol & 38.76 & 44.19 & 41.47 & - & - & - \\
\hline
\end{tabular}

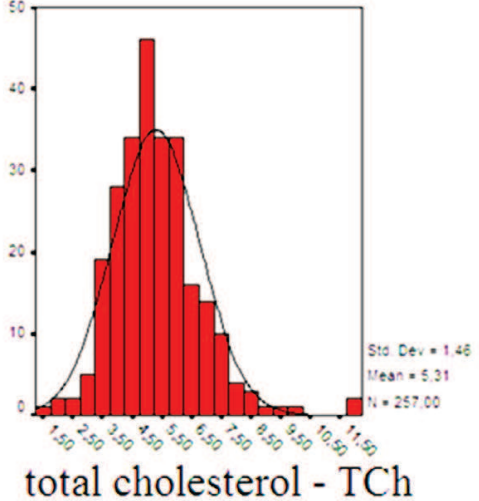

Fig. 1. Distribution of AIS patients according to total cholesterol values

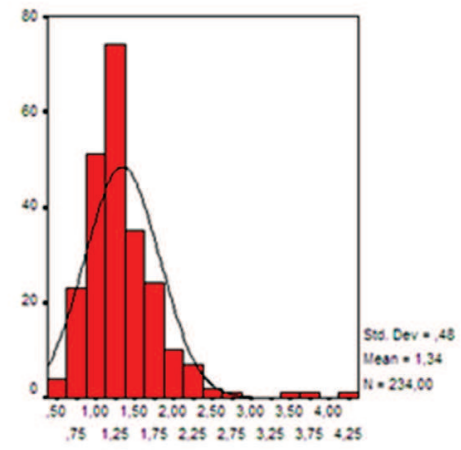

\section{HDL - cholesterol}

Fig. 3. Distribution of AIS patients according to HDLcholesterol values

ly elevated in our AIS patients. Its mean values are higher in the female patients who present with normal, decreased and increased LDL-cholesterol levels. The number and relative share of the male patients with increased and with decreased HDL-cholesterol values are higher than the number and relative share of the female ones. Our male AIS patients with normal triglyceride levels and our female AIS patients with reduced triglyceride levels considerably prevail.

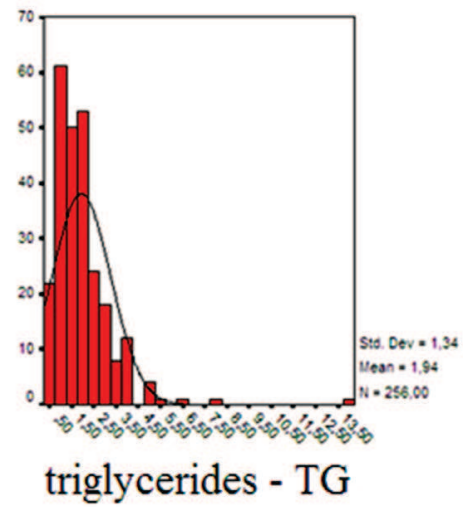

Fig. 2. Distribution of AIS patients according to triglyceride values

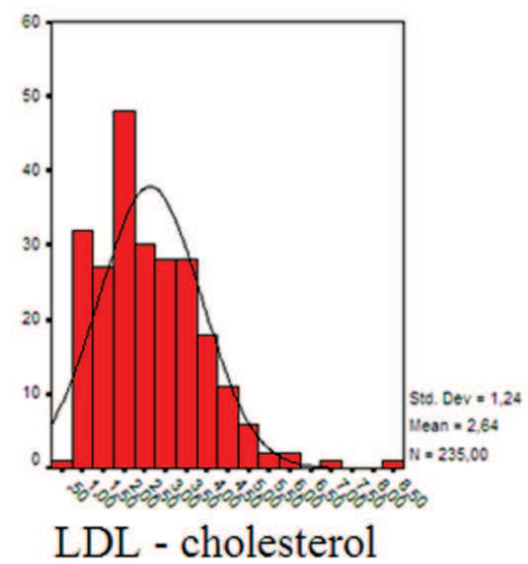

Fig. 4. Distribution of AIS patients according to LDLcholesterol values

Second come the females with elevated and the males with reduced triglyceride values.

\section{DISCUSSION}

Our results indicate the presence of hyperlipidemia such as enhanced total cholesterol and LDLcholesterol and dyslipidemia such as reduced triglycerides along with either increased, or decreased 
HDL-cholesterol in AIS patients at advanced age. They display the importance of some disturbances of lipid metabolism for the development of AIS in adult and elderly subjects. A plausible explanation of these findings consists in the relatively unhealthy life-style of the representatives of our contingent who, as a rule, do not undergo any regular laboratory examinations prior to the AIS accident. In most cases, there is no previous strict control and proper management of their, most often, slight or moderate cardiovascular pathology.

Because of the considerable scattering of the individual values of these parameters, however, the specific differences remain statistically non-significant ( $>0.05)$.

Recently, similar data were reported in the foreign literature available.

The influence of classic cardiovascular disease risk factors such as family history of these diseases, hypercholesterolemia, arterial hypertension, diabetes mellitus, overweight and obesity, on the development of a first IS in 250 consecutive patients was comprehensively examined (7). The prevalence of decreased HDL cholesterol levels $(\mathrm{p}<0.001)$ and elevated LDL cholesterol ones ( $\mathrm{p}=0.036)$ is significantly higher in IS patients than in controls. The decreased HDL cholesterol level $(\mathrm{p}<0.001)$ acts as independent risk factor for IS in young and middle-aged individuals (8).

The analysis of 3290 acute IS admissions at a regional stroke center in the USA shows higher mean plasma total cholesterol and higher blood circulating LDL levels $(\geq 100 \mathrm{mg} / \mathrm{dL})$ in all women, except South Asian ones, than in men and suggests that women may be at increased risk for IS large-artery atherosclerosis (9). Fasting and non-fasting triglycerides and HDL-cholesterol are associated with IS only when patients present with low levels of LDL-cholesterol (10). Higher on-admission serum fasting triglycerides predict less severe disability, reduced disability progression, and all-cause mortality in patients with AIS (11). By using multivariate logistic regression and propensity score-adjusted analyses, positive correlations between the serum levels of total cholesterol, LDL-cholesterol and HDL-cholesterol and the outcome in AIS patients are established (12). In the course of a prospective cohort study of 3
093 IS patients at a mean age of 66.8 years it is established that low HDL-cholesterol $(\leq 35 \mathrm{mg} / \mathrm{dL})$ at admission is associated with higher initial IS severity and poor clinical outcome during the follow-up in these patients (13).

AIS severity and initial total cholesterol levels independently influence the outcome in 374 out of 2 772 patients, or in $13.49 \%$ of the cases who are aged $>80$ years (14). Based on an observational study using data from the Get-With-The-Guidelines-Stroke Registry including 913436 patients with AIS or transient ischemic attack it is concluded that management of dyslipidemia in high-risk patients continues to be suboptimal as only one out of five such patients presented with optimal admission LDL values $<70 \mathrm{mg} / \mathrm{dL}$ (15). The analysis of 9940 AIS patients reveals baseline dyslipidemia in 5838 (in $58.73 \%$ of the cases) along with AIS recurrence in 2202 ones (in $22.15 \%$ of the cases) (16). The probability of this recurrence increases in patients with large-artery disease and dyslipidemia as compared with other AIS subtypes $(\mathrm{p}<0.013)$.

AIS is diagnosed in 73 out of 2620 Iranians aged $\geq 50$ years. Only among females, total cholesterol, LDL-cholesterol and non-HDL-cholesterol are independently related to increased risk of AIS (17). Among 31 AIS patients with more than 50\% stenosis proved by carotid Doppler sonography in Kathmandu, Nepal, 28 have hyperlipidemia (18).

Serum lipoprotein(a) levels are statistically significantly higher in 153 AIS patients in China than in 120 normal controls $(\mathrm{p}=0.001)$ (19). The multivariate analysis proves an increased risk of unfavourable outcome associated with lipoprotein(a) levels $(\mathrm{p}=0.001)$ after adjusting for possible confounders. Low total cholesterol $(<4.6 \mathrm{mmol} / \mathrm{L})$ is related to higher risk of death in AIS (20). Three-month, one-year and fiveyear survival rates are $100 \%, 98 \%$ and $84 \%$ in the patients with high total cholesterol but $92 \%, 87 \%$ and $57 \%$ in those with low one, respectively $(\mathrm{p}=0.0001)$.

\section{CONCLUSIONS}

A regular control of the common parameters of lipid metabolism and keeping-up the healthy lifestyle in adult asymptomatic individuals and in patients with already diagnosed cardiovascular diseases as well within the outpatient clinical practice 
Daniela Arabadzhieva, Zdravko Slavov, Ara Kaprelyan

could ensure a successful AIS prevention and its sufficient socio-economic effectiveness in our country.

\section{REFERENCES}

1. Bharosay A, Bharosay VV, Bandyopadhyay D, Sodani A, Varma M, Baruah H. Effect of lipid profile upon prognosis in ischemic and haemorrhagic cerebrovascular stroke. Indian J Clin Biochem. 2014;29(3):372-6.

2. Hsu CY, Hu GC, Chen YM, Chen CH, Hu YN. Predictors of short- and long-term mortality in firstever ischaemic older stroke patients. Australas J Ageing. 2013;32(4):229-32.

3. Lisak M, Demarin V, Trkanjec Z, Basić-Kes V. Hypertriglyceridemia as a possible independent risk factor for stroke. Acta Clin Croat. 2013;52(4):458-63.

4. Miyamoto N, Tanaka Y, Ueno Y, Kawamura M, Shimada Y, Tanaka R, et al. Demographic, clinical, and radiologic predictors of neurologic deterioration in patients with acute ischemic stroke. J Stroke Cerebrovasc Dis. 2013;22(3):205-10.

5. Rist PM, Buring JE, Kase CS, Ridker PM, Kurth T. Biomarkers and functional outcomes from ischaemic cerebral events in women: a prospective cohort study. Eur J Neurol. 2013;20(2):375-81.

6. Weng WC, Huang WY, Su FC, Chien YY, Wu CL, Lee $\mathrm{TH}$, et al. Less favorable neurological recovery after acute stroke in patients with hypercholesterolemia. Clin Neurol Neurosurg. 2013;115(8):1446-50.

7. Kastorini CM, Georgousopoulou E, Vemmos KN, Nikolaou V, Kantas D, Milionis HJ, et al. Comparative analysis of cardiovascular disease risk factors influencing nonfatal acute coronary syndrome and ischemic stroke. Am J Cardiol. 2013;112(3):349-54.

8. Supanc V, Sonicki Z, Vukasovic I, Solter VV, Zavoreo I,. Kes VB. The role of classic risk factors and prothrombotic factor gene mutations in ischemic stroke risk development in young and middle-aged individuals. J Stroke Cerebrovasc Dis. 2014;23(3):e171-6.

9. Gezmu T, Schneider D, Demissie K, Lin Y, Giordano C, Gizzi MS. Lipid profiles and ischemic stroke risk: variations by sex within racial/ethnic groups. Int J Womens Health. 2014;6:585-95.

10. Kim SJ, Park YG, Kim JH, Han YK, Cho HK, Bang OY. Plasma fasting and nonfasting triglycerides and high-density lipoprotein cholesterol in atherosclerotic stroke: different profiles according to low- density lipoprotein cholesterol. Atherosclerosis. 2012;223(2):463-7.

11. Pikija S, Trkulja V, Juvan L, Ivanec M, Dukši D. Higher on-admission serum triglycerides predict less severe disability and lower all-cause mortality after acute ischemic stroke. J Stroke Cerebrovasc Dis. 2013;22(7):e15-24.

12. $\mathrm{Xu} \mathrm{T}$, Zhang JT, Yang $M$, Zhang $\mathrm{H}$, Liu WQ, Kong $\mathrm{Y}$, et al. Dyslipidemia and outcome in patients with acute ischemic stroke. Biomed Environ Sci. 2014;27(2):106-10.

13. Yeh PS, Yang CM, Lin SH, Wang WM, Chen PS, Chao TH, et al. Low levels of high-density lipoprotein cholesterol in patients with atherosclerotic stroke: a prospective cohort study. Atherosclerosis. 2013;228(2):472-7.

14. Cha JK, Lim JH, Kim DH, Nah HW, Park HS, Choi JH, et al. Prognostic factors for long-term poor outcomes after acute ischemic stroke in very old age ( $>80$ years) patients: Total cholesterol level might differently influence long-term outcomes after acute ischemic stroke at ages above 80 years. Geriatr Gerontol Int. 2014 Dec 11. doi: 10.1111/ ggi.12419.

15. Saposnik G, Fonarow GC, Pan W, Liang L, Hernandez AF, Schwamm LH, et al.; AHA Get-withthe-Guidelines Stroke. Guideline-directed lowdensity lipoprotein management in high-risk patients with ischemic stroke: findings from Get with the Guidelines-Stroke 2003 to 2012. Stroke. 2014;45(11):3343-51.

16. Kumral E, Evyapan D, Gökçay F, Karaman B, Orman M. Association of baseline dyslipidemia with stroke recurrence within five-years after ischemic stroke. Int J Stroke. 2014;9 Suppl. A 100:119-26.

17. Tohidi M, Mohebi R, Cheraghi L, Hajsheikholeslami F, Aref S, Nouri S, et al. Lipid profile components and incident cerebrovascular events versus coronary heart disease; the result of 9 years followup in Tehran Lipid and Glucose Study. Clin Biochem. 2013;46(9):716-21.

18. Tuladhar AS, Shrestha A, Adhikari P, Joshi LN, Sharma GR. Carotid Doppler and lipid profile findings in ischemic stroke patients - a hospital based study. Nepal Med Coll J. 2013;15(2):98-101.

19. Zhang $W$, Zhang XA. Prognostic value of serum lipoprotein(a) levels in patients with acute ischemic stroke. Neuroreport. 2014;25(4):262-6. 
Serum lipid anomalies in adult patients with acute ischemic stroke

20. Markaki I, Nilsson U, Kostulas K, Sjöstrand C. High cholesterol levels are associated with improved long-term survival after acute ischemic stroke. J Stroke Cerebrovasc Dis. 2014;23(1):e47-53. 